\title{
Introduction to the HICSS-51 Software Development for Mobile Devices, Wearables, and the Internet-of-Things Minitrack
}

\author{
Tim A. Majchrzak \\ University of Agder, Kristiansand, Norway \\ Email: timam@uia.no
}

\author{
Tor-Morten Grønli \\ Westerdals, Oslo, Norway \\ Email: tmg@westerdals.no
}

Applications for mobile devices (apps) have facilitated the success of smartphones and tablets. By using apps, the multipurpose hardware of modern devices can be utilized to the full extent. Despite much progress with regard to development methods, software development kits, and frameworks, app development poses many challenges. This is even truer for novel mobile devices such as wearables and for the hardware that constitutes the Internet-of-Things (IoT). Compatibility, performance, battery-saving, security, and testing are only some of the issues that are mainly driven by the quality of the used software. A satisfying level of this quality in many cases is very hard to achieve.

Experiences and methods from classical software development can only be utilized to some degree. Moreover, the inherent challenges of the respective new devices ask for novel solutions. The above-sketched issues are worsened by the conditions that underlie development. New threads of research are needed to tackle issues, and to pave the way for better business producibility and an improved customer experience.

The minitrack extends the former Mobile App Development minitrack (of HICSS-49 [1] and HICSS-50 [2]) beyond the core app realm, which was limited to apps for devices for smartphones and tablets. It keeps the focus on software development but broadens the scope of applicable hardware to reflect the increasing interconnectedness. The minitrack thereby is devoted to the technological background of mobile computing while keeping an eye on business value, user experience, and domain-specific issues.

The minitrack will cover two sessions. The first session covers technical and formal papers:

1) Metamorphic Testing of Navigation Software: A Pilot Study with Google Maps by Joshua Brown, Zhi Quan Zhou, and Yang-Wai Chow

2) A Language-based Approach for Interoperability of IoT Platforms by Maurizio Gabbrielli, Saverio Giallorenzo, Ivan Lanese, and Stefano Pio Zingaro

3) Energy-Aware Scheduling of Conditional Task Graphs on NoC-Based MPSoCs by Umair Ullah Tariq, Hui $\mathrm{Wu}$, and Suhaimi Abd Ishak

The second session takes a look at advances in crossplatform app development:

1) Bridging the Gap: Investigating Device-Feature Ex- posure in Cross-Platform Development by Andreas Biørn-Hansen and Gheorghita Ghinea

2) Evaluating a Graphical Model-Driven Approach to Codeless Business App Development by Christoph Rieger

3) Progressive Web Apps: the Definite Approach to CrossPlatform Development? by Tim A. Majchrzak, Andreas Biørn-Hansen, and Tor-Morten Grønli

We are glad that we again had many helping hands. We are proud that all authors that submitted papers to our track got at least three - most even four - constructive reviews as well as an additional meta review. We think that we outperform many journals with this effort in giving authors advice, be they accepted or not. Therefore, we would like to thank (and explicate!) our program committee for their arduous work:

- Philippe Dugerdil, Geneva School of Business Administration

- Gheorghita Ghinea, Brunel University

- Adrian Holzer, Ecole Polytechnique Fédérale de Lausanne

- Shah Rukh Humayoun, University of Kaiserslautern

- Grace A. Lewis, Carnegie Mellon Software Engineering Institute

- Syed Atif Mehdi, University of Central Punjab

- Pietro Murano, Høgskolen i Oslo og Akershus

- Laura Po, University of Modena and Reggio Emilia

- Mohammad Tafiqur Rahman, University of Agder

- Christoph Rieger, University of Münster

- Rocco Raso, Universität des Saarlandes

- Sergio Ríos-Aguilar, International University of La Rioja (UNIR)

- Davide Rossi, University of Bologna

- Johannes Schobel, Ulm University

- Gabriele Taentzer, Philipps-Universität Marburg

- Tony Wasserman, Carnegie Mellon University Silicon Valley

- Burkhard Claus Wuensche, University of Auckland

- Stelios Xinogalos, University of Macedonia, Thessaloniki

\section{References}

[1] T. A. Majchrzak and H. Heitkötter, "Introduction to the mobile app development minitrack," in Proc. 49th HICSS. IEEE Computer Society, 2016, p. 5682.

[2] T. A. Majchrzak and T.-M. Grønli, "Introduction to the mobile app development minitrack," in Proc. 50th HICSS. AIS Electronic Library (AISeL), 2017, p. 6161. 